
Development

\title{
Regulatory Barriers to the Advancement of Precision Medicine
}

\section{David Pettitt, James Smith, Nick Meadows, Zeeshaan Arshad, Anna Schuh, David DiGiusto, Chas Bountra, Georg Holländer, Richard Barker \& David Brindley}

To cite this article: David Pettitt, James Smith, Nick Meadows, Zeeshaan Arshad, Anna Schuh, David DiGiusto, Chas Bountra, Georg Holländer, Richard Barker \& David Brindley (2016): Regulatory Barriers to the Advancement of Precision Medicine, Expert Review of Precision Medicine and Drug Development

To link to this article: http://dx.doi.org/10.1080/23808993.2016.1176526

Accepted author version posted online: 08 Apr 2016.

Submit your article to this journal $₫$

LII Article views: 1

Q View related articles $₫$

View Crossmark data $\nearrow$ 
Publisher: Taylor \& Francis

Journal: Expert Review of Precision M edicine and Drug Development

DOI: $10.1080 / 23808993.2016 .1176526$

\section{Expert Review: Regulatory Barriers to the Advancement of Precision Medicine}

Authors:

David Pettitt $^{1,2}$, James Smith ${ }^{1,3}$, Nick Meadows ${ }^{4}$, Zeeshaan Arshad ${ }^{5}$, Anna Schuh $^{6}$, David DiGiusto ${ }^{7}$, Chas Bountra $^{8}$, Georg Holländer ${ }^{2,9}$, Richard Barker ${ }^{1,10}$, David Brindley ${ }^{1,2,11-13}$

1. The Oxford - UCL Centre for the Advancement of Sustainable Medical Innovation (CASMI), The University of Oxford, Oxford, UK.

2. Department of Paediatrics, Medical Sciences Division, University of Oxford, UK.

3. Nuffield Department of Orthopedics, Rheumatology and Musculoskeletal Sciences, University of Oxford, Oxford, UK.

4. Kinapse, Wimbledon, London, UK.

5. School of Medicine, University of St. Andrews, St. Andrews, UK

6. Oxford National Institute of Health Research (NIHR), Biomedical Research Centre (BRC), Molecular Diagnostic Centre (MDC), Oxford University Hospitals, Oxford, UK.

7. Stem Cell and Cellular Therapeutics Operations at Stanford University Hospital and Clinic, Stanford, CA, USA.

8. Structural Genomics Consortium, Nuffield Department of Medicine, University of Oxford, Oxford, UK.

9. Weatherall Institute of Molecular Medicine, University of Oxford, John Radcliffe Hospital, Oxford, UK

10. Precision Medicine Catapult, London, UK.

11. Centre for Behavioral Medicine, UCL School of Pharmacy, University College London, London, UK.

12. Harvard Stem Cell Institute, Cambridge, MA, USA.

13. USCF-Stanford Center of Excellence in Regulatory Science and Innovation (CERSI), USA.

Corresponding authors: David Pettitt (david.pettitt@hertford.ox.ac.uk)

\section{Acknowledgements}

We express sincere thanks to the following organizations that have contributed to the CASMI Translational Stem Cell Consortium (CTSCC) as funding and events partners, without whom the consortium and the benefits it will bring to stem cell translation would be constrained: GE Healthcare, the Center for Commercialization of Regenerative Medicine (CCRM), Sartorius Stedim Biotech (formerly TAR Biosystems), Lonza, the California Institute for Regenerative Medicine (CIRM), the Strategies for Engineered Negligible Senescence (SENS) Research Foundation, UK Cell Therapy Catapult, NIH Centre for Regenerative Medicine, the New York Stem Cell Foundation (NYSCF), ThermoFisher Scientific, Eisai, Medipost (US), Medipost (Korea), Celgene, Roche and Oxford Biomedica. Author Brindley gratefully acknowledges personal funding from the Oxford Musculoskeletal National Institute for Health Research (NIHR) the Saïd Foundation, and the SENS Research Foundation. Authors Pettitt and Smith gratefully acknowledge support from the CASMI Translational Stem Cell Consortium (CTSCC). Smith also gratefully acknowledges support from the UK Medical Research Council and Prof Andrew Carr.

Review

\section{Regulatory Barriers to the Advancement of Precision Medicine}

Authors: 
David Pettitt ${ }^{1,2}$, James Smith ${ }^{1,3}$, Nick Meadows ${ }^{4}$, Zeeshaan Arshad ${ }^{5}$, Anna Schuh $^{6}$, David DiGiusto ${ }^{7}$, Chas Bountra $^{8}$, Georg Holländer ${ }^{2,9}$, Richard Barker ${ }^{1,10}$, David Brindley ${ }^{1,2,11-13}$

14. The Oxford - UCL Centre for the Advancement of Sustainable Medical Innovation (CASMI), The University of Oxford, Oxford, UK.

15. Department of Paediatrics, Medical Sciences Division, University of Oxford, UK.

16. Nuffield Department of Orthopedics, Rheumatology and Musculoskeletal Sciences, University of Oxford, Oxford, UK.

17. Kinapse, Wimbledon, London, UK.

18. School of Medicine, University of St. Andrews, St. Andrews, UK

19. Oxford National Institute of Health Research (NIHR), Biomedical Research Centre (BRC), Molecular Diagnostic Centre (MDC), Oxford University Hospitals, Oxford, UK.

20. Stem Cell and Cellular Therapeutics Operations at Stanford University Hospital and Clinic, Stanford, CA, USA.

21. Structural Genomics Consortium, Nuffield Department of Medicine, University of Oxford, Oxford, UK.

22. Weatherall Institute of Molecular Medicine, University of Oxford, John Radcliffe Hospital, Oxford, UK

23. Precision Medicine Catapult, London, UK.

24. Centre for Behavioral Medicine, UCL School of Pharmacy, University College London, London, UK.

25. Harvard Stem Cell Institute, Cambridge, MA, USA.

26. USCF-Stanford Center of Excellence in Regulatory Science and Innovation (CERSI), USA.

Corresponding authors: David Pettitt (david.pettitt@hertford.ox.ac.uk)

\section{Abstract}

Precision medicine utilizes tailored diagnostic, prognostic and therapeutic strategies based on an individual's molecular profile. Although it is gaining considerable traction and high-level political endorsement, it must overcome a number of translational hurdles, including regulatory barriers.

At the core of precision medicine lies diagnostic tests and devices, however the regulatory classification of such products varies on a global basis. Navigating these convoluted regulatory pathways can be challenging - exacerbated by asymmetric technological advancement and regulatory progression. Both the EU and US are attempting to address such issues and newer concerns relating to direct-to-consumer testing.

Flexible solutions are required to establish regulatory compliance across multiple countries and coordinated cross-collaboration initiatives need to empower technological development and globally harmonized regulation. The wider infrastructure, spanning beyond regulation, must also accommodate these changes and support subsequent clinical adoption, in order to firmly establish precision medicine in modern day medical practice.

1. Introduction 
The advent of precision medicine, also known as "personalized" medicine, whereby tailored diagnostic, prognostic and therapeutic strategies target an individual's molecular profile, is an exciting concept but one not without a number of scientific, translational, and clinical hurdles. A topic of growing interest across global scientific and medical communities, precision medicine now occupies high-level political endorsement - such as US President Obama's State of the Union Address, where he outlined the National Institutes of Health \$215 million Precision Medicine Initiative (PMI) in January 2015 [2] or the ' $21^{\text {st }}$ Century Cures Act[3]. Such enthusiasm and governmental support extends internationally - the United Kingdom's National Institute for Health Research (NIHR) for example, has outlined provisions for more meaningful implementation of precision medicine within the UK's National Health Service (NHS), including a dedicated Genomics England project, to sequence 100000 whole genomes by 2017 and help realize the potential this innovative field offers[4]. The UK government has also launched the Precision Medicine Catapult to catalyze the translation process.

The precision medicine space is progressing at a considerable rate. Currently, substantial biotechnological advances have been made in relation to genomic sequencing and accompanying therapies, patient characterization capabilities (spanning everything from genomic assays to computational biology), big data, and other '-omics' [2]. The list of 'omics' under study is growing rapidly, from epigenomics and transcriptomics to assess gene expression, to proteomics and metabolomics to map the metabolic status of the cell. Most recently, microbiomics has emerged, linking the co-habiting organisms in the body with the health of the human host. All of these techniques promise a much more profound understanding of the molecular basis of disease and hence of potential therapy.

In many respects, cancer is in the lead in this process. Oncogenic targets such as human epidermal growth factor (HER2) in breast cancer [5], EGFR mutations in lung adenocarcinoma [6] and BRAF mutations in melanoma are now well established in clinical practice[7]. This has been made a reality through the technological evolution of next-generation sequencing (NGS) to identify potentially targetable genomic events, and subsequently allowing clinical oncology to emerge as the vanguard of precision medicine. Cross-collaboration initiatives by international enterprises are helping empower 
technological development and progression, and are being driven by operations such as the International Cancer Genome Consortium (ICGC) (https://icgc.org), national collaborations such as The Lung Cancer Mutation Consortium (LCMC) (https://www.golcmc.com) and through key stakeholder engagement at a regional level to integrate precision medicine into current strategic clinical research platforms[6,8,9].

Precision medicine (see Figure 1) is often cited as "the right drug for the right patient at the right dose at the right time" [10] and generally involves the utilization of two or more products, such as a diagnostic test or device to optimize patient response to a particular therapeutic intervention. This approach could apply to a multitude of therapeutic agents, including chemical drugs, biologic products, human cellular and tissue products, gene therapies, drug-drug combinations and xenotransplantation products - all of which necessitate regulatory scrutiny during their development and subsequent clinical adoption. Forthcoming initiatives such as the PMI Cohort Program in the US, which builds on the achievements of the UK BioBank [https://www.ukbiobank.ac.uk], seek to extend precision medicine capabilities to all disease states [through the creation of a one million subject national research cohort]. Such data-intensive, broad population initiatives will continue to expand the regulatory arena to incorporate pertinent topics such as data sharing and privacy, electronic health records and health information technology. Data sharing and analytics is vital for the progress of precision medicine, but the issues that arise from patients facing severe diseases are quite different from those of the general population.

Of course, doctors have always sought to be as precise as possible in matching therapy with the patient, but the arrival of the molecular tools of modern precision medicine enables a complete transition from a "one-size-fits-all" intuitive approach. However this transition poses both practical and regulatory challenges. The requisite infrastructure must be devised and stabilized, and multiple stakeholders need to collaborate in order to surpass a number of translational barriers, including as 
the design of clinical studies, reimbursement policies and market access strategies. An essential component of this is the requirement for an apposite regulatory framework that safeguards patients whilst facilitating a streamlined approval process in order to achieve optimum patient benefit and economic sustainability. Presently, a number of regulatory barriers are impeding the precision medicine enterprise and reducing its clinical impact, in part due to concerns regarding evidentiary standards, navigating convoluted regulatory pathways and the lag between technological progression and accompanying regulation. Below we examine key regulatory barriers and issues pertaining to the translation and implementation of "personalized medicine", focusing predominantly on EU and US markets.

In order that innovative products and combinations for precision medicine are available for regulation, considerable coordination is required between non-regulatory entities [1]. This consists of a close interplay between academia and industry, and necessitates a harmonized approach to realize the scientific opportunities precision medicine provides (see Figure 2).

Figure 2: Scientific Opportunities in Precision Medicine

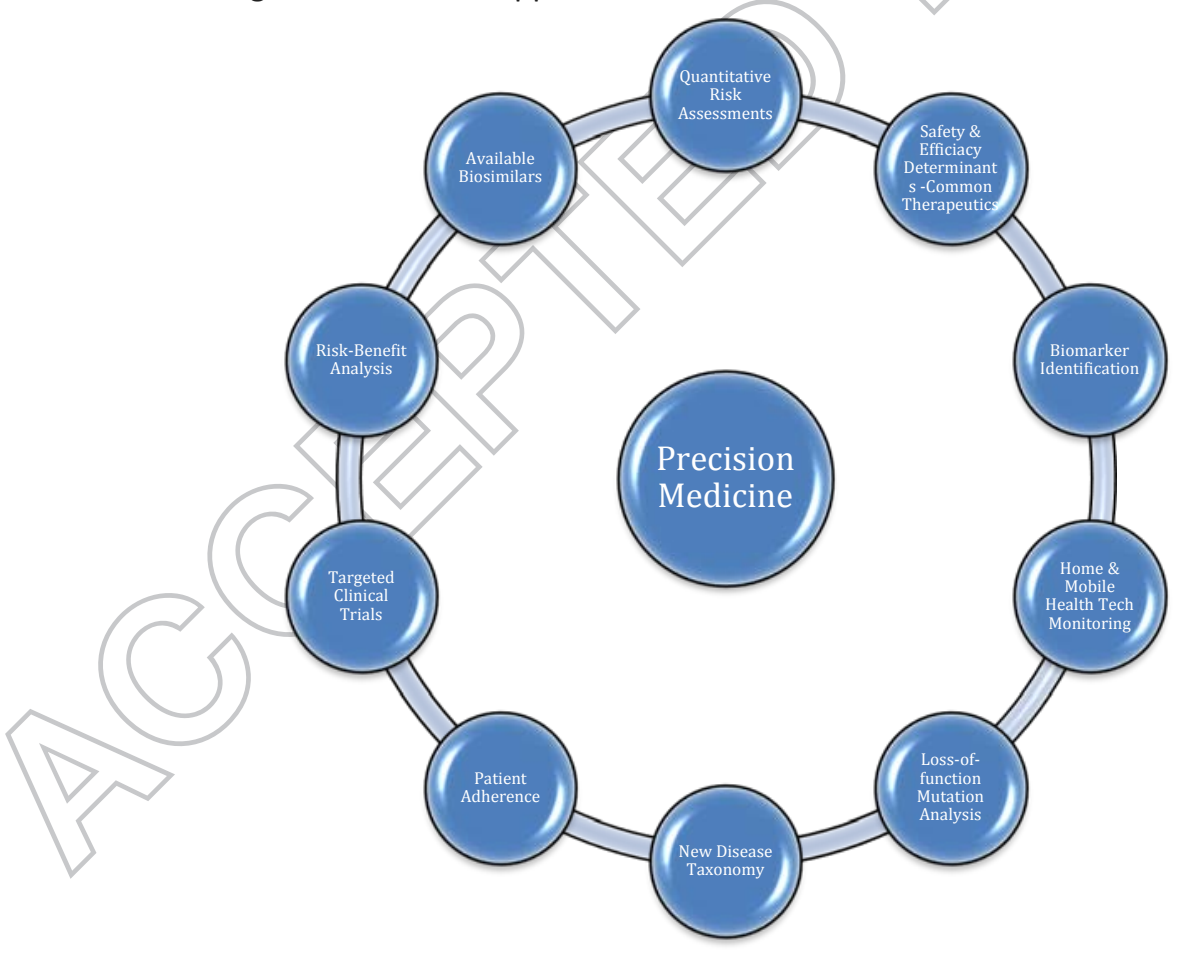

2. Diagnostic Devices in Precision Medicine 
At the heart of precision medicine, and central to its diagnostic foundation, lie diagnostic tests and devices, which measure or evaluate indicators of normal biological processes, pathogenic processes or therapeutic responses[11]. Diagnostics can be broadly categorized into in vitro or in vivo devices. In vitro diagnostic (IVD) devices encompass sequencing and assay technologies that examine the genetic basis of disease or biomarkers reflecting specific protein-level alterations. In vivo investigations consist of more conventional diagnostic imaging modalities such as $\mathrm{CT}, \mathrm{MRI}$ and PET, as well as electrocardiography and electroencephalography but progressively also encompass high-end imaging [10]. Regulatory classification of such products, and consequently their regulatory pathways, vary on a global basis (see Figure 3). There is also an important difference between the US situation, in which the targeting diagnostic and accompanying therapeutic are considered together, and the EU, in which different agencies make these decisions.

\subsection{US}

The US Food and Drug Administration (FDA) oversee the advancement, development and approval of both therapeutic and diagnostic agents. For marketing purposes, the US categorizes IVDs as either IVD kits or laboratory-developed tests (LDTs). IVDs may include kits such as the commercial Cystic Fibrosis Genotyping Assay (Abbott CE-IVD kit) [12] or standalone LDTs e.g. a KRAS test for colorectal cancer[13]. IVDs, diagnostic tests approved for commercial use, are subject to a risk-based classification (Class I to III) depending on whether they are considered low to moderate risk (Class I), moderate to high risk (Class II) or high risk (Class III) - with risk pertaining to how the results are used for medical decision-making. Class I IVDs are subject to General Controls comprising of basic provisions such as device registration, adverse event reporting and GMP practice requirements to ensure device safety and effectiveness. Class II IVDs, in addition to General Controls, are required to enforce Special Controls, which includes post-market surveillance activities,
Figure 3: Medical Device Legislation

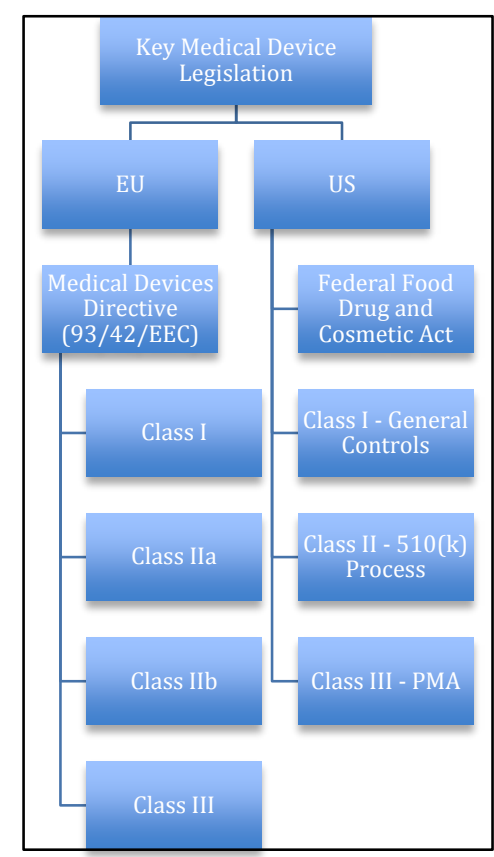


premarket data requirements and specific labeling requirements. Class II devices also require a $510(\mathrm{k})$

- a Premarket Notification - which is a premarket submission made to the FDA demonstrating safety, effectiveness and substantial equivalence to other legally marketed devices. Satisfying these requirements includes an assessment of bias, imprecision, analytical specificity and sensitivity, clinical samples and/or information, and rarely, prospective clinical studies. (Most Class I devices are exempt from such analysis).

Class III (high-risk) IVDs, must navigate the more comprehensive Premarket Approval (PMA) pathway. Submissions must include scientific evidence demonstrating safety and efficacy, manufacturing process and facility review outcomes and clinical studies that identify surrogate endpoints and establish device performance[14]. Prospective clinical studies are, of course, extremely expensive and may necessitate financial support from the manufacturer of the accompanying therapy.

\section{Table 1: US Classification of Clinical Laboratory Testing}

\begin{tabular}{|c|c|}
\hline Test & Regulation \\
\hline In Vitro Diagnostics (IVD) & Food, Drug and Cosmetic Act (FD\&C Act) \\
\hline $\begin{array}{l}\text { Analyte Specific Reagent } \\
\qquad \text { (ASR) }\end{array}$ & $\begin{array}{c}\text { Clinical laboratory responsible for quality } \\
\text { and analytical validity }\end{array}$ \\
\hline $\begin{array}{c}\text { Laboratory Developed } \\
\text { Test (LDT) }\end{array}$ & $\begin{array}{l}\text { No regulatory approval - to be used } \\
\text { within the laboratory }\end{array}$ \\
\hline
\end{tabular}

LDTs comprise protocols or kits designed, manufactured and originally intended for use in stand-alone laboratories. Unlike IVDs, LDTs were historically exempt from pre-market FDA review due to their classification as low-risk tests. However, reflecting advancements in both technology, complexity and application, and the increasing realization that incorrect results represented real risks for patients, the FDA advanced a draft guidance in October 2014 relating to LDTs in an attempt to risk-stratify them, reduce misdiagnoses and align them with IVDs (which are subject to tighter regulatory controls)(see Table 1) [15]. LDTs are currently subject to less stringent regulation, largely due to their relative simplicity when they were first introduced to the market. Under the CLIA (Clinical Laboratory 
Improvement Amendments) of 1988, laboratories performing 'waived' tests are subject to minimal regulation and laboratories performing moderate or high complexity tests are subject to specific laboratory standards governing areas such as certification, quality \& control, and inspections - in the EU, this governance filters down to member-state level and may account for differences in quality perspectives. Performing clinical laboratory testing at a CLIA certificated clinical laboratory does offer some advantages - notably recognized reimbursement for the process. Oncotype Dx (Genomic Health, Redwood City, CA, USA) for example is a widely performed 21-gene assay that predicts therapeutic response in early-stage oestrogen receptor positive $(E R+)$ breast cancers and is reimbursed by a number of health insurance payers [15].

In general, LDTs require less in the way of development costs in comparison to regulated equivalents, which undermines the commercial attractiveness of the diagnostics space [16]. However, it should be noted that initial investments in clinical trial testing to demonstrate clinical utility [and meet regulatory requirements] might be of considerable value - particularly if regulatory requirements vary geographically.

The FDA intends to reengineer this system and align the regulatory standards of LDTs with those of IVDs - risk-stratifying LDT kits into low, medium or high risk (Class I to III respectively) categories. This will require low risk LDT manufacturers, like their IVD kit equivalents, to submit information concerning test registration, listing and adverse events to regulators. Similarly, evidentiary requirements are elevated for tests deemed higher-risk; class II LDTs must go via the 510(k)/premarket notification and class III LDTs will have to meet the requirements for PMA [17]. The current and proposed pathways are outlined in Table 2 . These regulatory improvements are likely to discourage the misuse of LDTs and improve patient safety. However the more stringent regulation may present difficulties for manufacturers, and stifle the current pace of translation. Even for IVD kits, the current PMA review process displays several limitations; including the absence of a "gold standard" for benchmarking, appropriately defined minimum performance standards and the impact of potential bias secondary to study methodology. If these regulatory barriers are not mitigated, LDT developers will face similar hurdles to those experienced by IVD kit producers. 
Table 2: Comparison of the current US IVD regulatory framework and the proposed LDT regulatory framework $[14,18]$

FDA - Federal Food, Drug, and Cosmetic Act*

\begin{tabular}{|c|c|c|c|}
\hline \multicolumn{4}{|c|}{ FDA - Federal Food, Drug, and Cosmetic Act* } \\
\hline \multicolumn{2}{|c|}{ In Vitro Diagnostic [IVD] Kits } & \multicolumn{2}{|c|}{ Laboratory Developed Tests [LDT] } \\
\hline Framework & Requirements & $\begin{array}{l}\text { Proposed } \\
\text { Framework }\end{array}$ & Requirements \\
\hline \multirow[t]{2}{*}{ Class I } & General Controls & \multirow{2}{*}{$\begin{array}{l}\text { Class I: } \\
\text { Low risk }\end{array}$} & \multirow[b]{2}{*}{$\begin{array}{ll}\text { - } & \text { Registration } \\
\text { - } & \text { Device listing } \\
\text { - } & \text { Adverse event reporting } \\
\text { - } & \text { Exempt from quality system } \\
& \text { regulations }\end{array}$} \\
\hline & $\begin{array}{ll}\text { - } & \text { Registration } \\
\text { - } & \text { Device listing } \\
\text { - } & \text { AE reporting } \\
\text { - } & \text { GMP } \\
\text { - } & 510[\mathrm{k}] \\
\end{array}$ & & \\
\hline \multirow[t]{2}{*}{ Class II } & Special Controls & \multirow{2}{*}{$\begin{array}{c}\text { Class II: } \\
\text { Medium risk }\end{array}$} & \multirow[b]{2}{*}{$\begin{array}{l}\text { Begin reporting adverse events } \\
\text { to FDA within six months of the } \\
\text { guidance being finalized } \\
\text { - } \quad \text { Premarket review [premarket } \\
\text { notification or } 510[\mathrm{k}] \\
\text { Meet medical device cGMP }\end{array}$} \\
\hline & $\begin{array}{l}\text { - Performance standards } \\
\text { - Postmarket surveillance } \\
\text { - Patient registries } \\
\text { - } \text { Special labeling } \\
\text { requirements } \\
\text { - } \quad \text { Premarket data } \\
\text { - } 510[\mathrm{k}]\end{array}$ & & \\
\hline \multirow[t]{2}{*}{ Class III } & PMA & \multirow{2}{*}{$\begin{array}{l}\text { Class III: } \\
\text { High risk }\end{array}$} & \multirow{2}{*}{$\begin{array}{l}\text { - } \text { As Class II } \\
\text { - PMA }\end{array}$} \\
\hline & $\begin{array}{ll}\text { - } & \text { As Class II } \\
\text { - } & \text { Clinical studies }\end{array}$ & & \\
\hline
\end{tabular}

* IVDs may also be biological products subject to Section 351 of the Public Health Service Act.

The increasing technological complexity of such tests makes the standardization of evidence and regulatory evaluation difficult, particularly for those looking to enter global markets where a number of distinct regulatory systems exist. Diagnostic tests also employ different business models to therapeutics - diagnostics manufacturers typically lack the financing and resources available to larger (bio)pharmaceutical organizations.

Whole genome sequencing represents a particular regulatory challenge, as it comprises of sample handling and preparation, the sequencing itself, the 'calling' of gene variants and the clinical interpretation of the results. In 2015 the FDA began a process of consultation on regulations to cover some, not all, of the steps. We will return to this question later[19]. 
In the EU, IVDs are governed by the Directive of In Vitro Diagnostic Medical Devices (98/79/EC) - one

of three principal EU directives regulating medical devices (see

Box 1) [20]. In contrast to the US, European approval, in the form

of a 'CE mark' can be sought through any of a number of 'notified

bodies' (rather then the European Medicines Agency that

regulates drugs).

Similarly to the US, the EU is currently undergoing a period of

Box 1: EU Medical Device Directives

- Medical Devices Directive [93/42/EEC]

- Directive of Active Implantable Medical Devices $[90 / 385 /$ EEC]

- $\quad$ Directive of In Vitro Diagnostic Medical Devices $[98 / 79 / E C]$

regulatory transition with regards to medical devices and IVDs, hastened by high profile scandals such as the Poly Implant Prostheses (PIP) or metal-on-metal joint replacements reference [21,22]. Directive 98/79/EC provides the current regulatory framework for placing IVDs on the EU market but stands to be replaced with new IVD regulation, which will be subject to a phased implementation over the next five years[23]. Again, this adopts a 'risk-based' classification system and will comprise of four risk classes (A-lowest to D-highest) with the intention of safety-enhancing the existing approval system[22].

Key elements of the proposed regulation include the involvement of the notified bodies (for Class B, C \& D devices), performance of evaluation reports [covering scientific, analytical and clinical dimensions] and market surveillance data

(whereby an electronic system will capture serious incidents and events that may alter initial risk-benefit analyses).

Notified bodies exist at member state

level - there is no pan-European

regulatory body with global authority.

Class A devices - those deemed lowest

risk - will be subject to a conformity

assessment, which will be the sole

\section{Case Study 1: Ovasure}

- Ovasure diagnostic test was thought to predict the onset of ovarian cancer by measuring six blood proteins.

- Developed by researchers at Yale University with trials being carried out by LabCorp

- These markers included prolactin, leptin, insulin-like growth factor II, macrophage inhibitory factor, osteopontin and CA-125 [used to measure disease progression in ovarian cancer[54]]

- Classified as a LTD [Laboratory Developed Test] instead of a medical device for regulatory purposes

- Reached market without FDA approval and was commercially available in 2008

- Concerns led to the FDA requesting its removal from market several months after release[32,55], and included:

o Incorrect prevalence rate used to calculate the products positive predictive value

- A number of false positive results [leading to unnecessary ovarian removal] 
responsibility of the manufacturer (with the exception of devices intended for self testing, near-

patient testing or sterilization procedures) $[23,24]$. The proposed regulation will also help address the current lack of evidentiary requirements for lower class devices concerning utility.

For precision medicine to be truly realized, a clearer recognition of outstanding limitations in the existing regulatory systems is required. By definition, precision medicines need to be trialed on smaller populations and development and approval pathways must utilize innovative clinical trial designs. The process must also be made shorter and more flexible [25]. Findings by Cancer Research UK estimated that between 2003 and 2007, time taken for study approval increased by 65\% coupled with a $75 \%$ increase in administration-related costs [26]. Novel clinical trials utilizing adaptive, basket or umbrella designs, which target select patient groups, may help alleviate barriers associated with conventional clinical trials and their associated regulation, and streamline the pathway to clinical adoption $[26,27]$. They will also enable pharmacogenomic or other 'omic' testing to be more widely implemented in therapeutic clinical trials. Most of the existing FDA approved biomarkers deal with avoiding safety issues rather than selecting for superior response: this balance needs to shift over time.

\section{Companion Diagnostics [CDX]}

\begin{tabular}{c|l}
\multicolumn{2}{c}{ Table 3: Examples of FDA-approved Cancer } \\
Biomarkers
\end{tabular}

BTA: bladder tumour-associated antigen; c-Kit: stem cell growth factor receptor; EGFR: epidermal growth factor receptor, GIST: gastrointestinal stromal tumour, K-RAS: v-Ki-ras2 Kirsten rat sarcoma viral oncogene homologue; PSA: prostate-specific antigen.

By identifying, measuring and evaluating specific patient characteristics such as gene expression or 'omics' (e.g. microbiomics, proteomics, metabolomics), predictive biomarkers (see Table 3[28]) enable clinicians to personalize treatment and optimize patient outcomes (see Figure 4) [29] [30]. 
This helps reduce variation in response to treatment - in the US for example; it is estimated that the ten highest grossing drugs are only effective in between $4 \%$ and $25 \%$ of the patients who are prescribed them - and can also aid with safety and efficacy profiling [10]. Spurred on by these statistics, the 'omics' space is seeing exponential technological and scientific progression and there are now over 150 FDA-approved drugs incorporating genomic information in their prescriptive guidelines to-date. However, progress has not been smooth: the dangers of misconstruing results provided by consumer genomics companies have been highlighted in a number of previously conducted studies [31]. Tests such as Ovasure (see Case Study 1), which was designed to detect earlystage ovarian cancer, was beleaguered with high false-positive rates, which resulted in a number of avoidable oophorectomies[32]. This phenomenon of 'imprecision medicine' remains a concern for regulators and must be fully addressed to avoid patient harm [27].

Figure 4: A Genomics-based Approach to Medicine[29]

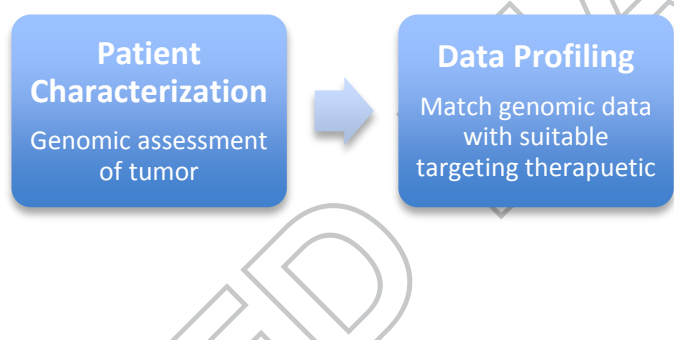

Therapeutic Algorithm

Personalized

anticancer-cocktail

for clinical delivery

Precision medicine has typically utilized CDx tests, which are defined by the FDA (2014) as "a medical device, often an in vitro device, which provides information that is essential for the safe and effective use of a corresponding drug or biological product". They have proven to be indispensable commodities in the shift from empirical to precision driven healthcare models - reflected in their 2014 market valuation of $\$ 3.14$ billion[33]and an annual market growth in the region of $25 \%[34]$. CDx tests have substantially aided the quest for therapy by establishing and exploiting an individual's molecular profile and enabling accurate alignment of an efficacious therapeutic agent - which ultimately maximizes patient responsiveness and overall health outcomes $[10,35]$.

Although the EU has not formally adopted a definition of CDx, the Council of EU Ministers' proposal concerning new IVD regulation infers that their definition is likely to reflect that of the FDA's. Presently, and in contrast to the US, the EU does not have a coordinated regulatory mechanism that 
jointly evaluates both therapeutics and CDx. The European Medicines Agency [EMA] does not regulate diagnostics; National Competent Authorities manage trials and approval, whilst evaluation and certification is conducted by Notified Bodies (which may actually be perceived as a less burdensome process for applicants).

For therapeutics, new drugs seeking EU-wide marketing approval obtain authorization via the European Medicinal Agency's (EMA) Committee for Medicinal Products for Human Use (CHMP), whereby marketing authorization applications (MAA) detailing product quality, safety and efficacy are evaluated in accordance with Regulation (EC) No 726/2004 and Directive 2001/83/EC[36]. (Under a little-used decentralized pathway, parties may submit MAA to the National Competent Authority of a single member state). CDx on the other hand are independently regulated under the In Vitro Diagnostic Directive 98/79/EC and attract a CE (Conformité Européene) mark if the product fulfills apposite legal, safety and quality criteria - as declared by the product manufacturer. The CE mark is granted via a number of European Commission-accredited independent organizations (notified bodies). The relationship between a CDx and its corresponding drug only becomes apparent through drug labeling requirements; drugs that use CDx and are seeking marketing approval under either of the aforementioned pathways are required to declare appropriate IVD CDx devices on their labeling. A concern here is that is that any directive 98/79/EC compliant IVD CDx can be paired with a drug even though efficacy and validity properties may vary between individual devices. Consequently, discrepancies in a clinical impact, detection ability and ultimately, patient impact, may prevail[36].

These uncoordinated regulatory pathways in the EU, comprising independent therapeutic and CDx processes, is a source of considerable uncertainty for both drug and device manufacturers seeking market entry, and furthermore, could distract from the purpose of precision medicine - to accurately

identify the most effective treatment for a particular patient. In an attempt to aid both drug developers and device manufacturers, the EU is currently transitioning through a period of regulatory overhaul and seeks to adopt an umbrella classification system based on risk - aligning the EU's regulatory mechanisms alongside those of the US. The proposed amendments intend to establish a pre-market review process for the majority of IVDs, which will hopefully ease the "co-regulation" burden, in addition to seeing CDx qualify as Class C devices - a significantly higher level of regulation 
compared to conventional "self-certification" by manufacturers[37]. Implementation of the modifications will commence with a phased approach from 2016 onwards and spanning at least five years. However, existing problems are likely to persist unless a single pan-European body, such as the EMA, is better utilized as the overarching authority. Short of coordinated regulation, it has been suggested that joint scientific advice be made available to the therapeutic and CDx manufacturers[38].

The concept of contemporaneous approval, whereby new molecular entities (NMEs) or new biological entities (NBEs) and CDx can be approved via a single application to the FDA is a major difference between EU and US regulatory pathways [10]. Under the auspices of the FDA, IVDs are reviewed by the Center for Devices and Radiological Health (CDRH) and therapeutics by either the Center for Drug Evaluation and Research (CDER) or the Center for Biologics Evaluation and Research (CBER). This intrinsically linked approach requires substantial co-ordination during the early development phases but also serves as an incentive for collaboration and co-development between pharmaceutical manufacturers and diagnostic developers[35]. It facilitates knowledge sharing at the pharmaceuticaldeveloper interface and helps streamline the requisite regulatory pathway[16]. However, this model also poses significant challenges for the stakeholders involved, notably the pharmaceutical organizations, who, if developing drugs alongside $\mathrm{CDx}$, will rely on clinical trial designs and regulatory flexibility to co-ordinate the alignment of device and drug development timelines.

Precision medicine stands to make a potentially significant impact on global health in the long-term. This however presents a number of regulatory barriers for all stakeholders involved. Even beyond the increasing complexity of devices, diagnostics and therapeutics, there is a paucity of regulatory

harmonization internationally. The commercial significance of this is likely to be further compounded by strong growth rates in emerging markets.

Regulatory convergence is needed even among developed markets. Japan for example - an established market with a generally highly regulated pharmaceutical and medical device industry currently lacks both a formal LDT regulatory definition and a regulated LDT reimbursement system. 
This has inevitably allowed for "home-brew assays" to be developed and unapproved devices to be used in clinical practice[15]. Unfortunately, the inherent complexity of precision medicine means regulators are unable to simply streamline or modify existing frameworks, but must act on a fundamental need to develop entire quality management systems.

A shift away from the "one-size-fits-all" paradigm reflected in conventional regulatory practice to a future where single diagnostic tests or complex panels may be employed to direct clinicians towards an array of highly effective therapeutics requires early regulatory engagement and harmonization on an international scale. Global partnerships such as the Global Coalition for Regulatory Science Research (GCRSR), which aims to foster regulatory consensus amongst international partners and integrate novel technologies into regulatory decision-making, aims to help overcome challenges concerning validation, transparency and an application's "fitness for purpose" [1]. Such partnerships may also help address a number of financial and intellectual property issues concerning diagnostics for example, the first CDx for Amgen's (Thousand Oaks, California, US) Vectibix (panitumumab) warranted investment for studies to show clinical utility concerning KRAS gene detection, and once proven, the kit could be replicated by "home-brew" competitors - discouraging first movers in the market place[39].

Case Study - Herceptin

- Most widely cited example of the development of personalized medicine is Trastuzumab [Herceptin ${ }^{\circledR}$ ] - approved in 1998

- Roche/Genentech was the first company to successfully use the "therapy-test" co-development model, bringing Herceptin ${ }^{\circledR}$ (Trastuzumab, Genentech-Roche, San Francisco, USA) to the market in 1998, [with simultaneous approval of Dako's immunohistochemistry assay HercepTest ${ }^{\mathrm{TM}}$ (Dako, Agilent Technologies, Santa Clara, USA), targeting HER2 overexpression in advanced breast cancer. [36]

- First precision therapy for the treatment of HER2-positive metastatic breast cancers

- Reduced recurrence by $52 \%$ in combination with chemotherapy[10]

- The "development pipeline" for Herceptin biosimilars also appears promising, with approximately 54 currently in development (see Chart 1 below). 


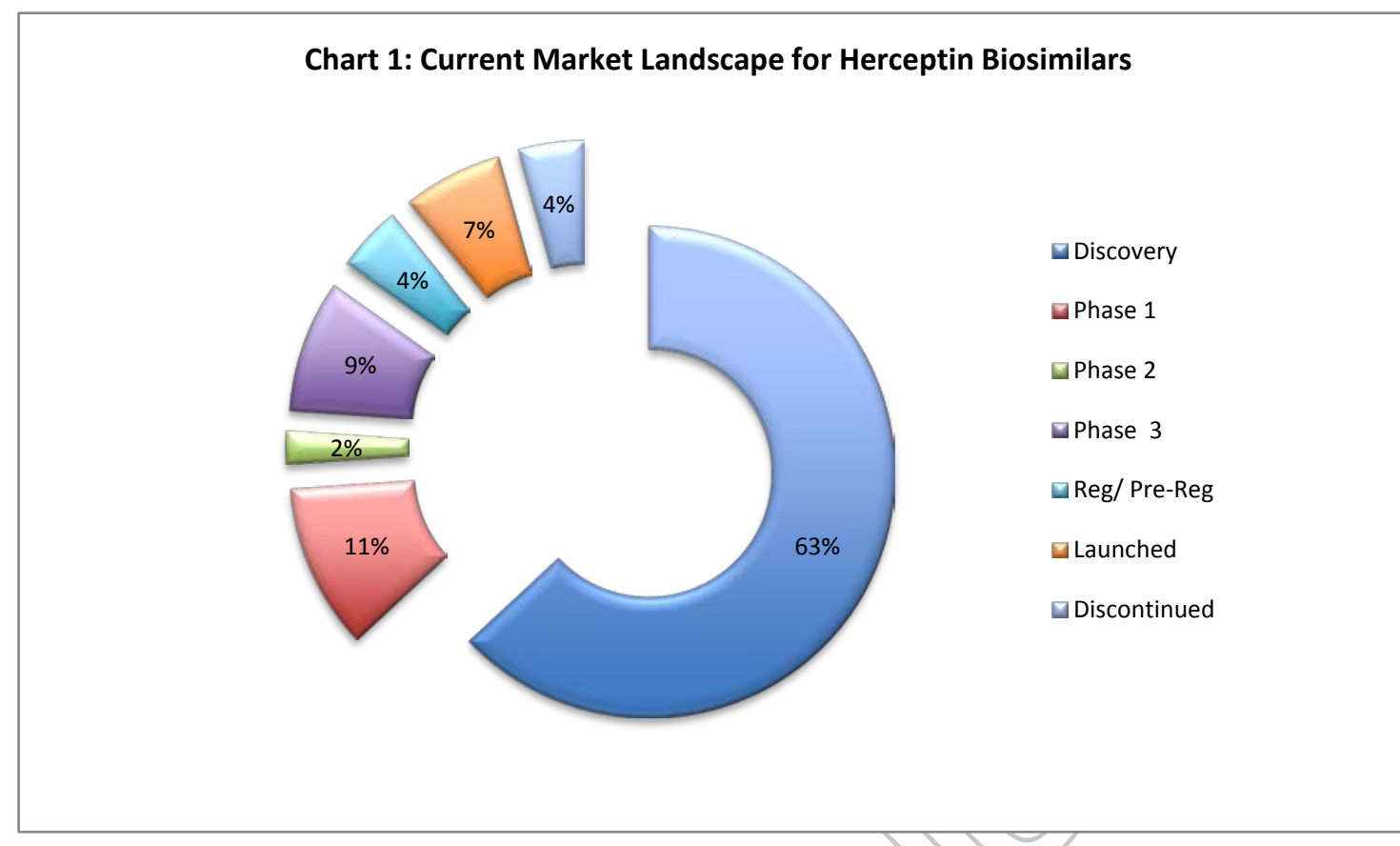

4. Biomarkers \& Genomics

Genomic sequencing, which is a specialist technology that is able to effectively identify genomic variants compared to a reference sequence, has evolved considerably since the first human genome was sequenced in 2003 as part of the Human Genome Project. Cost per genome sequencing by next generation sequencing (NGS) is now available for under $\$ 1300$ - a figure that is envisaged to further decrease as technology progresses[40](https://www.genome.gov/sequencingcosts/). As NGS becomes more mainstream and transitions from research to a more prominent position in daily clinical practice, novel regulatory initiatives are required to parallel such activity.

The US Centers for Disease Control and Prevention (CDC) has addressed this need and established a work group to ensure that NGS tests comply with existing regulatory and quality standards[41]. The

Next Generation Sequencing: Standardization of Clinical Testing (Nex-StoCT) Work Group serves as an (

effective platform for key stakeholders to formulate quality assurance guidelines and facilitate the use of NGS-sequence results in clinical practice - ensuring that test results are both reliable and beneficial to patients[41]. Authors have also discussed working towards 'good genomic practice' (GGP) - the field's equivalent of good laboratory practice (GLP) and good manufacturing practice (GMP) to facilitate its development[42]. A number of approved single-gene tests are now available in 
routine clinical practice for mutations including KRAS, BRAF and EGFR. The "trailblazer" for FDA marketing authorization of such tests was Illumina's MiSeqDx (see Case Study 2) - approved in late 2013 to detect DNA mutations in cystic fibrosis [43]. Its marketing authorization pathway also served as a valuable insight into the approval process for other manufacturers and outlined what the FDA expected in terms of validity and verification data. Concern however remains that a number of unregulated genetic tests are being sold directly to consumers and this problem appears to be proliferating[44].

Regulators must therefore address these issues; ensuring that product development is effectively regulated to maintain patient and consumer safety without discouraging innovation.

\section{Case Study 2: Illumina's MiSeqDx}

- Received CE marking in June 2013

- FDA-clearance as a class II device in November 2013 [510[k]]

- FDA made the device and substantially similar devices exempt from the premarket notification requirements.

- FDA also approved Illumina's cystic fibrosis carrier screening assay

\section{Key data requirements:}

- Accuracy data (specimen types \& nucleic acid types)

- Sequence data

- Comparison to reference sequences

- Precision studies

- Cross-contamination \& interfering substances

Key Challenges:

- False positives \& subsequent quality control to test performance

- Lack of standardization

- Orthogonal validation

- No. of mutations requiring clinical validation

Regulation of such a complex market is challenging. Reliance on conventional clinical trial data can translate into high development costs. In some cases there is an insufficient number of patients for conventional trials, and in others the ethics of randomizing potentially terminal patients into placebo arms when there appears to be a medicine targeted to their specific disease has been questioned. Regulating the analysis of huge numbers of gene variants also poses difficulties and governing bodies may therefore need to engage external expertise into their current systems e.g. the FDA could collaborate with the College of American Pathologists [who already routinely conduct laboratory quality certifications] [17].

The capabilities of NGS are likely to evolve as genomic analysis is paired with proteomics, metabolomics and microbiomics, amongst other technological advancements, to allow cross-platform profiling. Novel clinical trial structures will need to facilitate the adoption of precision medicine into 
standard clinical applications by shifting proof-of-concept studies into early-phase clinical trials and subsequently, the clinic[6].

5. Personalized testing: Clinicians and Patients

As the results of these complex analyses are integrated into a molecular profile of a specific patient's disease, we will see the emergence of algorithms to assist the clinician in his/her interpretation. These clinical decision support tools may themselves raise regulatory questions if they become too instructive. Conversely, we are seeing considerable market growth for direct-to-consumer genetic testing, (DTC-GT) claiming to profile an array of diseases including cancer, Parkinson's disease and diabetes[45]. Easily accessible via online marketplaces, clinicians are being asked to interpret results from tests where the underlying scientific integrity, validity and clinical utility are questionable[46]. Despite efforts from both UK and US regulators, global regulatory enforcement for internet-based industries remains exceptionally challenging[47]. Although some tests have been reported as potentially clinically useful amongst healthcare professionals[48,49], stringent quality criteria and ethical standards are not guaranteed - and nor is responsible use[46]. Such challenges have come to light in a number of high-profile cases, including 23andMe (Mountain View, California, US) [50] - a US based genetic testing and analytics company who in November 2013 were instructed by the FDA to discontinue their Personal Genome Service[51][on the basis that false results may lead to unnecessary interventions] [49]. Although current evidence concerning the risk of DTC-GT is uncertain[51], the market is forecast to grow to over $\$ 230$ million (USD) by 2018[49] and regulators must therefore maintain vigilance and a sector presence - for example by enforcing the provision of genetic counseling, ensuring delivery models include access to intermediary expertise and discrediting misleading claims.

Most fundamentally, precision medicine ushers in the 'molecular taxonomy of disease'. Diseases will increasingly be defined in terms of their molecular pathology rather than their symptoms, their gross pathology and/or the part of the body in which they appear ('non-small cell lung cancer'). Patient populations for trials and treatment will therefore need to be specified in terms of this new 
taxonomy, and regulation, reimbursement and clinical practice will each need to adapt over time to this new reality.

6. Conclusion

Although EU and US regulators are in the process of shaping new legislation to deal with some of the novel features of precision medicine for their respective healthcare systems, a number of critical milestones are yet to be reached before precision medicine constitutes standard clinical practice. A major determinant will be the degree to which international communities can harmonize regulatory policies and help clinically useful technologies become more accessible on a globalscale, and indeed, support those developers seeking to access the global market - which has implications from a commercialization perspective in terms of return on income (ROI) and also in progressing the concept of precision medicine as a whole. Before harmonization can be achieved on an international scale, regulatory frameworks must be optimized at a regional level — while the US already permits contemporaneous diagnostic and approval applications via the FDA, in Europe, the EMA is currently detached from diagnostic regulators, and greater convergence is needed over the long-term. The most recent EU amendments largely bypass this issue and utilization of the EMA as a single panEuropean regulatory body therefore appears unlikely[16]. Both EU and US regulatory bodies, alongside transnational research communities, need to provide guidance on how best to address issues of harmonization at both national and international levels.

Technological advances will continue to challenge conventional regulatory requirements. Modified clinical trial models such as the adaptive design employed in the "I-SPY 2" trial, utilizing biomarkers to streamline the testing of breast cancer drugs[52], will become more prominent. The new European concept of adaptive pathways[53]is underpinned by the precision medicine concept, as it anticipates conditional approval for patient sub-populations selected by biomarkers.

In parallel with regulatory challenges, there are a number of other common barriers that must be overcome in the precision medicine space. Historically, the substantial profit margins of large pharmaceutical companies are derived from the 'blockbuster' model, under which - in addition to 
patients that receive benefit - patients take drugs with little to no effect. Narrower markets challenge the business model if precision medicine replaces conventional prescribing practice. Conversely, such changes may facilitate wider implementation of value-based pricing models, which would allow pharmaceutical companies to charge more for advanced treatments and their associated clinical outcomes.

Closely interlinked with sustainable product development is the mechanism of reimbursement, which remains poorly defined with respect to novel CDx modalities, particularly in regions such as the US, where multiple payers are at play and recent reimbursement changes have been unhelpful. Cohesive regulatory policies, clear recognition of clinical utility and 'risk-sharing' reimbursement models will help reduce payer uncertainty and ultimately help increase the uptake of new technologies. The impact such technologies will have on clinical practice and the clinical workforce will inevitably constitute a shift in culture and require considerable up-skilling and training. Further regulatory considerations and quality assured management systems will also be required to address significant increases in data collection, sharing and use-at-scale as growth beyond non-cancer applications gains momentum.

Despite the present regulatory barriers, efforts are being made on both national and global stages to translate the potential of precision medicine into clinical practice and patient benefit. Regulators have reassuringly recognized the potential dangers of unregulated diagnostics and emphasized the importance of patient safety within their most recent amendments. They must now continue to recognize the importance of integrating complex facets into a unified system. Precision medicine needs redesigned evidentiary requirements and support by appropriate utilization of Big Data, early recognition of clinical utility and substantiated by reputable business models that deliver financial sustainability. Precision medicine presents a unique opportunity to revolutionize the medical landscape and in keeping with Hippocrates' ethos, 'it's far more important to know what person the disease has than what disease the person has'. 


\section{Expert Commentary}

The field of precision medicine is advancing at an encouraging rate, strengthened by both national level political endorsement and international appetite for an array of technologically advanced applications that offer clinical benefit to patients. As with the adoption of any advanced technology, it is essential that a robust platform be in place to help realize the opportunities available. Looking specifically at regulation within the US and EU, considerable progress has been made concerning the safe and effective translation of precision medicine from basic science research to a multitude of clinical applications, and now regulators must focus on streamlining current systems to facilitate market entry for requisite stakeholders. This will require timely transition from conventional regulatory practices to appropriately modified mechanisms that precision medicine warrants. In parallel to modifications pertaining to individual regulatory jurisdictions, regulators must also consider the impact of their actions on a global scale and seek to harmonize regulatory practice establishing regulatory compliance across multiple countries. Accompanying such changes, academic and commercial stakeholders must also recognize the importance of designing and implementing appropriate reimbursement systems to ultimately facilitate clinical adoption, which will be challenging - particularly where a complex blend of private and public payers are involved. For successful clinical adoption, modified clinical training and practice must reflect the changing landscape and early, broad exposure of applications to clinicians and allied healthcare professionals will aid familiarization and accelerate subsequent provision to patients. A flexible, multi-faceted approach that increasingly extends beyond regulatory considerations is therefore required to firmly establish this complex yet highly promising field in modern day medical practice.

8. Five-Year View

As the field of precision medicine expands and infiltrates further into mainstream medical practice, a number of developments will take place. Companion diagnostics are likely to become more common and may serve as potential differentiators in the biosimilars landscape. The market trends and the rational for diagnostics (which is currently somewhat unclear) will become more evident as the market matures - and this will inevitably see a growing emphasis placed on intellectual property protection. In terms of reimbursement, value based pricing mechanisms may emerge as the most favorable of the reimbursement platforms currently available due to its potential suitability for public funders, private payers and pharmaceutical companies (who can levy higher charges for more advanced therapeutics). The next decade is likely to be critical in the development of novel business and regulatory models amenable to the provision of precision medicine and will likely be directed by 
the next successful iteration of commercialized cell or gene therapy products or diagnostic

applications. Globally, there appears to be supportive networks in place that currently incorporate key academic, clinical and commercial stakeholders. Combining these with increased investor confidence and flexible regulatory systems will help precision medicine move to the forefront of patient care.

\section{Key Issues}

- Presently, the level of global regulatory harmonization and standardization is insufficient regulators must collaborate and work towards greater regulatory coordination in order to facilitate the adoption of precision medicine and address regional and global imbalances with access to technological applications.

- Outside of the US, there are deficiencies with regards to systems facilitating contemporaneous diagnostic and approval applications.

- Current business models require further optimization and adaptation(s) to incorporate increasingly narrower markets.

- Further regulation is required to accommodate the substantial increases in data collection, data protection and data use-at-scale.

- Global efforts are required to ensure patient safety by mitigating unregulated diagnostics markets - particularly genetic tests that are currently being sold direct-to-consumers (DTC).

- As precision medicine becomes more mainstream, clinical decision making algorithms are likely to become more widely adopted to support healthcare professionals. Their implementation and subsequent integration must be accompanied by appropriate regulatory systems

- Novel clinical trial designs should be optimized for the translation of precision medicine applications.

- Hybridization initiatives are needed as -omics'-based technological platforms (e.g. genomics, proteomics and metabolomics) become more widely utilized.

- Ethical considerations, especially relating to publicly accessible genomic sequencing services and ownership of data, are likely to become increasingly prevalent.

\section{Disclaimer}

This article represents the authors' individual opinions and may not necessarily represent the viewpoints of their employers.

\section{Acknowledgements}

We express sincere thanks to the following organizations that have contributed to the CASMI Translational Stem Cell Consortium (CTSCC) as funding and events partners, without whom the consortium and the benefits it will bring to stem cell translation would be constrained: GE Healthcare, the Center for Commercialization of Regenerative Medicine (CCRM), Sartorius Stedim Biotech (formerly TAP Biosystems), Lonza, the California Institute for Regenerative Medicine (CIRM), the Strategies for Engineered Negligible Senescence (SENS) Research Foundation, UK Cell Therapy Catapult, NIH Centre for Regenerative Medicine, the New York Stem Cell Foundation (NYSCF), ThermoFisher Scientific, Eisai, Medipost (US), Medipost (Korea), Celgene, Roche and Oxford Biomedica..

\section{Declaration of interest}


D Brindley gratefully acknowledges personal funding from the Oxford Musculoskeletal National Institute for Health Research (NIHR) the Saïd Foundation, and the SENS Research Foundation. D Brindley is a stockholder in Translation Ventures Ltd. (Charlbury, Oxfordshire, UK) and IP Asset Ventures Ltd. (Oxford, Oxfordshire, UK), companies that among other services provide cell therapy biomanufacturing, regulatory, and financial advice to pharmaceutical clients. J Smith is a consultant with IP Asset Ventures Ltd. Brindley also is subject to the CFA Institute's codes, standards, and guidelines, so he must stress that this piece is provided for academic interest only and must not be construed in any way as an investment recommendation. Additionally, at time of publication, D Brindley and the organizations with which he is affiliated may or may not have agreed and/or pending funding commitments from the organizations named herein. D Pettitt and J Smith gratefully acknowledge support from the CASMI Translational Stem Cell Consortium (CTSCC). J Smith also gratefully acknowledges support from the UK Medical Research Council and Prof Andrew Carr. The authors have no other relevant affiliations or financial involvement with any organization or entity with a financial interest in or financial conflict with the subject matter or materials discussed in the manuscript apart from those disclosed.

\section{References}

Papers of special note have been highlighted as:

* of interest

$* *$ of considerable interest

[1] Tong W, Ostroff S, Blais B, Silva P, Dubuc M, Healy M, et al. Genomics in the land of regulatory science. Regulatory Toxicology and Pharmacology 2015;72[1]:102-106.

[2] Collins FS, Varmus H. A new initiative on precision medicine. N Engl J Med 2015;372[9]:793-795.**

**Useful overview of precision medicine.

[3] Brindley DA, Arshad Z, Luo D, Dopson S, Hollander G, Frost S, et al. 21st Century Cures Act: An Act of Cure or Diagnosis? Rejuvenation research 2015;18[4]:295-298.

[4] Marx V. The DNA of a nation. Nature 2015;524[7566]:503-505.*

*Useful overview of the implications of genomics and current global initiatives.

[5] Swain SM, Baselga J, Kim S, Ro J, Semiglazov V, Campone M, et al. Pertuzumab, trastuzumab, and docetaxel in HER2-positive metastatic breast cancer. N Engl J Med 2015;372[8]:724-734.

[6] Buettner R, Wolf J, Thomas RK. Lessons Learned From Lung Cancer Genomics: The Emerging Concept of Individualized Diagnostics and Treatment. Journal of Clinical Oncology 2013 May 20;31[15]:1858-1865.

[7] McArthur GA, Chapman PB, Robert C, Larkin J, Haanen JB, Dummer R, et al. Safety and efficacy of vemurafenib in BRAF V600E and BRAF V600K mutation-positive melanoma [BRIM-3]: extended follow-up of a phase 3, randomised, open-label study. The lancet oncology 2014;15[3]:323-332.

[8] Arnedos M, Vicier C, Loi S, Lefebvre C, Michiels S, Bonnefoi H, et al. Precision medicine for metastatic breast cancer [mdash] limitations and solutions. Nature Reviews Clinical Oncology 2015.

[9] Hyman DM, Solit DB, Arcila ME, Cheng DT, Sabbatini P, Baselga J, et al. Precision medicine at Memorial Sloan Kettering Cancer Center: clinical next-generation sequencing enabling nextgeneration targeted therapy trials. Drug Discov Today 2015. 
[10] Tsourounis M, Stuart J, Pignato W, Toscani M, Barone J. Current Trends in Personalized Medicine and Companion Diagnostics A Summary From the DIA Meeting on Personalized Medicine and Companion Diagnostics. Therapeutic Innovation \& Regulatory Science 2015:2168479015570330.

[11] NIH. Precision Medicine Initiative Cohort Program. 2015; Available at: https://www.nih.gov/precision-medicine-initiative-cohort-program.

[12] Loukas YL, Thodi G, Molou E, Georgiou V, Dotsikas Y, Schulpis KH. Clinical diagnostic NextGeneration sequencing: The case of CFTR carrier screening. Scand J Clin Lab Invest 2015;75[5]:374381.

[13] Halling KC, Schrijver I, Persons DL. Test verification and validation for molecular diagnostic assays. Arch Pathol Lab Med 2012;136[1]:11-13.

[14] FDA. In Vitro Companion Diagnostic Devices. 2014.

[15] Tazawa Y. Perspective for the development of companion diagnostics and regulatory landscape to encourage personalized medicine in Japan. Breast Cancer 2015:1-5.

[16] Meadows N, Morrison A, Brindley D, Schuh A, Barker R. An evaluation of regulatory and commercial barriers to stratified medicine development and adoption. The pharmacogenomics journal 2014.*

* Overview of regulatory (and commercial barriers), including scientific and financial perspectives

[17] Hwang TJ, Lehmann LS, Kesselheim AS. Precision medicine and the FDA's draft guidance on laboratory-developed tests. Nat Biotechnol 2015;33[5]:449-451.

[18] FDA. Framework for regulatory oversight of laboratory developed tests [LDTs] — draft guidance. 2014.

[19] FDA. Medical Devices. 2015[February].

[20] Mason S. European regulation of medical devices. The Textbook of Pharmaceutical Medicine, 7th Edition 2013:418-434.

[21] Greco C. The Poly Implant Prothèse breast prostheses scandal: Embodied risk and social suffering. Soc Sci Med 2015;147:150-157.

[22] Milmo S. Regulation of Medical Devices and Companion Diagnostics. Pharm Technol 2015;39[8].

[23] European Commission. In Vitro Diagnostic Medical Devices: Directive 98/79/EC. 1998.

[24] European Commission. Use of '-omics' technologies in the development of personalised medicine . 2013.

[25] Barker R. A flexible blueprint for the future of drug development. The Lancet 2010;375[9712]:357-359.

[26] Mirnezami R, Nicholson J, Darzi A. Preparing for precision medicine. N Engl J Med 2012;366[6]:489-491.

[27] Schork NJ. Personalized medicine: Time for one-person trials. Nature 2015 Apr 30;520[7549]:609611. 
[28] Ludwig JA, Weinstein JN. Biomarkers in cancer staging, prognosis and treatment selection. Nature Reviews Cancer 2005;5[11]:845-856.

[29] Garraway LA, Verweij J, Ballman KV. Precision Oncology: An Overview. Journal of Clinical Oncology 2013 May 20;31[15]:1803-1805.

[30] Malottki K, Biswas M, Deeks JJ, Riley RD, Craddock C, Johnson P, et al. Stratified medicine in European Medicines Agency licensing: a systematic review of predictive biomarkers. BMJ Open 2014 Jan 27;4[1]:e004188-2013-004188.

[31] Green RC, Farahany NA. The FDA is overcautious on consumer genomics. Nature 2014;505[7483]:286-287.

[32] Buchen L. Cancer: Missing the mark. Nature 2011 Mar 24;471[7339]:428-432.

[33] Dimond PF. Companion Diagnostics for Cancer: Will NGS Play a Role? Clinical OMICs 2015;2[11]:8-11.*

*Details key considerations relating to the use of NGS

[34] Waldman SA, Terzic A. Companion diagnostics at the intersection of personalized medicine and healthcare delivery. Biomark Med 2015;9[1]:1-3.

[35] Luo D, Smith JA, Meadows NA, Manescu K, Bure K, Davies B, et al. A Quantitative Assessment of Factors Affecting the Technological Development and Adoption of Companion Diagnostics. Frontiers in Genetics 2015;6:357.

[36] Akhmetov I, Ramaswamy R, Akhmetov I, Thimmaraju PK. Market access advancements and challenges in "drug-companion diagnostic test" co-development in Europe. Journal of personalized medicine 2015;5[2]:213-228.

[37] European Commission. EC proposed regulation 2012/0267, In Vitro Diagnostic Medical Devices . 2012; Available at: http://ec.europa.eu/growth/sectors/medical-devices/regulatory-

framework/revision/index en.htm.

[38] Academy of Medical Sciences. Realising the Potential of Stratified Medicine. 2013.

[39] Schmidt C. Larger companies dominate cancer companion diagnostic approvals. Nat Biotechnol 2011;29[11]:955-957.

[40] Evans BJ, Burke W, Jarvik GP. The FDA and genomic tests-getting regulation right. N Engl J Med 2015.**

**Useful overview of key US regulatory legislation and its applications to genomic testing.

[41] Gargis AS, Kalman L, Berry MW, Bick DP, Dimmock DP, Hambuch T, et al. Assuring the quality of next-generation sequencing in clinical laboratory practice. Nat Biotechnol 2012;30[11]:1033-1036.

[42] Barker RW, Brindley DA, Schuh A. Establish good genomic practice to guide medicine forward. Nat Med 2013;19[5]:530-530.

[43] Sheridan C. Milestone approval lifts Illumina's NGS from research into clinic. Nat Biotechnol 2014;32[2]:111-112. 
[44] Jain K. Textbook of Personalized Medicine. Second ed. New York: Springer Science; 2015.*

*In-depth overview of precision medicine.

[45] Parthasarathy S. Assessing the social impact of direct-to-consumer genetic testing: understanding sociotechnical architectures. Genetics in Medicine 2010;12[9]:544-547.

[46] Hauskeller C. Direct to consumer genetic testing. BMJ 2011 Apr 21;342:d2317.

[47] Hogarth S, Javitt G, Melzer D. The current landscape for direct-to-consumer genetic testing: legal, ethical, and policy issues. Annu.Rev.Genomics Hum.Genet. 2008;9:161-182.

[48] Giovanni MA, Fickie MR, Lehmann LS, Green RC, Meckley LM, Veenstra D, et al. Health-care referrals from direct-to-consumer genetic testing. Genetic Testing and Molecular Biomarkers 2010;14[6]:817-819.

[49] Covolo L, Rubinelli S, Ceretti E, Gelatti U. Internet-Based Direct-to-Consumer Genetic Testing: A Systematic Review. J Med Internet Res 2015 Dec 14;17[12]:e279.

[50] www.23andme.com. Available at: www.23andme.com. Accessed March, 2016.

[51] Delaney S, Christman M. Direct-to-consumer genetic testing: Perspectives on its value in healthcare. Clinical Pharmacology \& Therapeutics 2016;99[2]:146-148.

[52] Barker A, Sigman C, Kelloff G, Hylton N, Berry D, Esserman L. I-SPY 2: An Adaptive Breast Cancer Trial Design in the Setting of Neoadjuvant Chemotherapy. Clinical Pharmacology \& Therapeutics 2009;86[1]:97-100.

[53] Eichler H, Baird L, Barker R, Bloechl-Daum B, Børlum-Kristensen F, Brown J, et al. From adaptive licensing to adaptive pathways: Delivering a flexible life-span approach to bring new drugs to patients. Clinical Pharmacology \& Therapeutics 2015;97[3]:234-246.

[54] Nolen BM, Lokshin AE. Biomarker testing for ovarian cancer: clinical utility of multiplex assays. Molecular diagnosis \& therapy 2013;17[3]:139-146.

[55] Levenson D. The trouble with Ovasure. Am J Med Genet A 2009 Mar;149A[3]:viii-ix. 